Cuadernos de Filología Clásica. Estudios Latinos

ISSN: 1131-9062

http://dx.doi.org/10.5209/cfcl.71529

\title{
Los disjuntos en las construcciones nominalizadas de evento en latín
}

\author{
Eveling Garzón Fontalvo ${ }^{1}$
}

Recibido: 8 de junio de 2020 / Aceptado: 3 de septiembre de 2020

Resumen. En latín los nombres deverbales que refieren la actividad o estado descrito por sus verbos base pueden dar lugar a construcciones nominalizadas de evento. Estas construcciones no solo son capaces de reflejar tanto los argumentos como los adjuntos de sus respectivas predicaciones verbales base $(\S 1)$, sino que también documentan elementos que aportan información relevante para el acto de comunicación en sí mismo y que, desde esta perspectiva, pueden identificarse como disjuntos o expresiones meta-comunicativas ( $(2)$. A partir de aquí, el objetivo de este artículo es doble. Por una parte, busca describir cuáles son los contenidos semánticos expresados por los disjuntos que aparecen dentro de las predicaciones nominalizadas (§ 3$)$. Por otra, pretende establecer qué restricciones sufren estos elementos en su transformación desde las predicaciones verbales a las estructuras nominales, al tiempo que intenta ofrecer una explicación para tales limitaciones $(\S 4)$.

Palabras clave: nominalización deverbal; nombres de evento; construcciones nominalizadas de evento; disjuntos.

\section{[en] The disjuncts in event nominal constructions in Latin}

Abstract. In Latin, the verbal nouns that refer to the activity or state described by their base verbs can give rise to event nominal constructions. These constructions are not only capable of reflecting both arguments and adjuncts of their respective underlying verbal predications $(\S 1)$, but also document elements that provide relevant information for the act of communication itself and can be therefore identified as disjuncts or meta-communicative expressions $(\S 2)$. In this vein, the purpose of this article is twofold. On the one hand, it seeks to describe what kind of semantic contents are expressed by the disjuncts within the nominalised constructions $(\S 3)$. On the other, it questions whether these elements suffer restrictions in their transformation from verbal predications to nominal structures and, as the case may be, it aims to offer an explanation of these limitations (§ 4).

Keywords: verbal nominalisation; event nouns; event nominal constructions; disjuncts.

Sumario. 1. Introducción. 2. Otros elementos dentro de las Construcciones Nominalizadas de Evento: los disjuntos. 3. Tipo de contenido semántico expresado por los disjuntos. 4. Restricciones de los disjuntos en su paso a las Construcciones Nominalizadas de Evento. 5. Conclusiones. 6. Referencias bibliográficas.

Cómo citar: Garzón Fontalvo, E. «Los disjuntos en las construcciones nominalizadas de evento en latín», Cuad. Filol. Clás. Estud. Lat. 40.1 (2020), 11-24.

\footnotetext{
Departamento de Filología Clásica, Universidad Autónoma de Madrid.

Correo electrónico: eveling.garzon@uam.es
} 


\section{Introducción}

La nominalización deverbal, esto es, el proceso mediante el cual un verbo se transforma funcionalmente en nombre, es uno de los fenómenos más interesantes y reveladores en cuanto a la relación existente entre ambas categorías de palabras. Para llevar a cabo un proceso de nominalización deverbal el latín cuenta, entre otras, con una estrategia denominada nominalización por formación morfológica o derivación, que consiste en aplicar un sufijo nominalizador a una base verbal ${ }^{2}$. Los ejemplos de (1)-(4) presentan diversos procesos regulares de derivación a través de los sufijos -tio, -tus, -tor y -culum.

(1) conspiro 'conspirar'> conspiratio 'conspiración'

aborior 'abortar' > abortus 'aborto'

(2) creo 'crear' > creator 'creador, fundador'

pacifico 'negociar la paz' > pacificator 'mediador, pacificador'

(3) aedifico 'edificar, construir' > aedificatio 'edificación, construcción'3 cogito 'pensar, reflexionar'> cogitatio 'pensamiento, reflexión'

(4) teneo 'tener' > tenaculum 'tenaza'

cubo 'estar echado, dormir' > cubiculum 'dormitorio'

A través de un proceso de derivación morfológica, se pueden generar distintos tipos de nombres: o bien un nombre que refiera el evento descrito por el verbo, así en (1), o bien un nombre que incorpore en su significado uno de los constituyentes del verbo. Dentro de este último grupo, un nombre puede hacer referencia al agente, como el de crear y negociar la paz en (2); al constituyente que se identifica con el tema y que se produce como resultado del evento, sea un objeto típico o cognado, caso de 'edificación' y 'pensamiento' en (3); al instrumento o al lugar, como 'tenaza' y 'dormitorio' en (4); aunque también existen otras posibilidades (Comrie \& Thompson $2007^{2}$ [1985], 334).

Los nombres de los ejemplos (1)-(4) presentan distintos grados de nominali$\operatorname{dad}^{4}$ de acuerdo con su capacidad para conservar y actualizar la estructura semántica, es decir, la complementación obligatoria, de sus respectivos verbos base $\mathrm{s}^{5}$. Constituyen el foco de este estudio nombres como conspiratio o abortus -denominados aquí nombres de evento- que designan la actividad o estado descrito por

\footnotetext{
Entre los estudios generales más relevantes sobre los nombres deverbales en latín se encuentran Boegel (1902), Benveniste (1975² [1948]), Rosén (1981, 1983), Pultrová (2011) y Spevak (2015). Para un estado de la cuestión actualizado, véase Garzón (2018).

3 Es importante señalar que un nombre como aedificatio puede designar no solo el resultado de la acción de edificar, sino también la acción misma. Sobre la distinción entre las lecturas eventivas y resultativas de los nombres deverbales en latín, cf. Rosén (1981), Garzón (2018) y, de manera tangencial, Spevak (2015).

4 Véase Hopper \& Thompson (1985) en relación con la iconicidad que manifiestan las categorías Nombre y Verbo desde una perspectiva tipológica.

5 Esta capacidad de algunos nombres deverbales se ha abordado desde diferentes perspectivas teóricas con carácter general y también específico para determinadas lenguas; cf., entre otros muchos trabajos, Grimshaw (1990), Picallo (1991, 1999), Hout (1991), Alexiadou (2001), Bisetto \& Melloni (2007), Sleeman \& Brito (2010a, 2010b) y Koptjevskaja-Tamm (2012).
} 
sus verbos base y que, además, pueden reflejar todo el esquema argumental de estos últimos ${ }^{6}$.

Esta particular capacidad se ilustra a través de las construcciones (5) y (6) con el nombre populatio 'devastación'. En el primero de estos pasajes, los nombres murum formicarumque que aparecen en genitivo se corresponden con el Sujeto de una predicación verbal como (5'). Por su parte, en el segundo, el genitivo de (6), agri, se identifica con el constituyente Objeto de una construcción del tipo (6') ${ }^{7}$.

(5) nam ea res a populatione murum formicarumque frumenta defendit («pues esto defiende el grano de la devastación de los ratones y de las hormigas», Colum.2.19.1 $)^{8}$.

(5') mures et formicae [frumenta] populantur.

(6) Omnis ira belli ad populationem agri uertit («Toda la ira se encausó hacia la devastación del territorio», Liv.2.62.3).

(6’) [Is] agrum populatur.

La documentación de un esquema de complementación por parte de un nombre de evento, como en los ejemplos (5) y (6), da lugar a lo que a partir de ahora denominaré una Construcción Nominalizada de Evento $(C N E)^{9}$. Desde esta perspectiva, es claro que las CNE remiten a una construcción verbal del tipo de (5') y (6'). Como se observa, en ellas la codificación sintáctica que presentan los constituyentes obligatorios de las predicaciones verbales es diferente, puesto que el cambio de categoría (de verbo a nombre) implica modificaciones ${ }^{10}$.

Del mismo modo, también pueden aparecer en una CNE elementos que funcionan como adjuntos o satélites dentro de las predicaciones verbales. En este sentido, pueden observarse las construcciones de (7) y ( $\left.7^{\prime}\right)$ : en (7), la CNE codifica mediante el adjetivo frequentem, que determina a locationem, una información adjunta de Frecuencia que en su potencial predicación verbal (7') sería un adjunto expresado por medio de un adverbio como frequenter o saepe ${ }^{11}$.

Siguiendo la clasificación de Lyons (1977), Spevak (2014, 182 ss.) ha realizado un estudio sobre las expansiones obligatorias de algunos sustantivos de primer y segundo orden en latín.

7 Koptjevskaja-Tamm (2012) ha propuesto una clasificación en la que establece un continuum entre las construcciones nominales cuyos argumentos conservan las marcas de sus correspondientes predicaciones verbales base y aquellas en las que sus argumentos son asimilados a la estructura del sintagma nominal.

8 Las traducciones que se ofrecen en este estudio son propias; no obstante, con el fin de limitar al máximo los errores de interpretación que se producen al trabajar con pasajes descontextualizados y de corroborar las lecturas aquí propuestas, se han contrastado con otras traducciones españolas y, en algunos casos, con traducciones francesas, italianas e inglesas.

$9 \quad$ Un panorama general sobre los tipos de constituyentes que pueden funcionar como argumentos adnominales con nombres de evento (verbal nouns) se ofrece en Pinkster (2015, 1037-1047).

10 Aunque existe una correspondencia entre los argumentos de las predicaciones verbales base y los de las CNE, hay una diferencia significativa entre ambos tipos de construcciones: «The difference resides in the fact that the terms associated with a nominalized verbal predicate are much more likely not to be expressed than is the case with non-nominalized verbal predicates» (Mackenzie 1985, 32).

11 Givón $(2001,25)$ reconoce la conversión de adverbios en adjetivos como un tipo de ajuste entre las predicaciones verbales finitas y su paso a las construcciones nominales prototípicas. 
(7) Ita certe mea fert opinio rem malam esse frequentem locationem fundi («Yo opino que, sin duda, el frecuente arrendamiento del campo es mala cosa», Colum.1.7.3).

(7') frequenter/saepe fundum locat.

Para dar cuenta de la variedad de adjuntos que se documentan dentro las CNE, se muestran los pasajes de (8)-(10):

(8) Verum suburbanis locis, ubi a matre pulli non exiguis pretiis ueneunt, probanda est aestiua educatio («Pero en los lugares cercanos a las ciudades, donde los pollos recién salidos de la madre se venden a precios no módicos, ha de intentarse la cría veraniega», Colum.8.5.9).

(9) Comitatus muliebris plus tacito fletu quam ulla uox mouebat («La comitiva de mujeres con su llanto callado conmovía más que cualquier palabra», Liv.3.47.4).

(10) Quid loquar quanta ratio in bestiis ad perpetuam conseruationem earum generis appareat? («¿Qué decir de cuánta razón se manifiesta en las bestias con vistas a la perpetua conservación de su especie?», Cic.Nat.Deor.2.128).

Como se observa, en estos ejemplos los adjetivos aestiua, tacito y perpetuam aportan información sobre el Tiempo (8), el Modo (9) y la Duración (10) de los eventos descritos por los nombres de evento a los que acompañan (cf. educatio, fletu y conseruationem, respectivamente $)^{12}$.

A la luz de los ejemplos (5)-(10), está claro que las CNE en latín tienen la posibilidad de reflejar tanto los argumentos como los adjuntos o satélites de sus correspondientes predicaciones verbales base. ¿Pero son estos los únicos elementos que se documentan en este tipo de construcciones?

Este artículo se propone tres objetivos: i) identificar qué otros componentes, además de los argumentos y adjuntos de los verbos base, pueden incluirse en las CNE; ii) describir cuáles son los contenidos semánticos expresados por estos; y iii) establecer si estos componentes sufren restricciones en su transformación desde las predicaciones verbales a las CNE.

Para ello, se ha optado por trabajar con un corpus base que contempla todos los nombres de evento en -tio y -tus (y en los respectivos alomorfos -sio y -sus) presentes en la obra completa de Plauto, los libros I y II de Philippicae de Cicerón, los libros I-IV de Ab urbe condita de Livio y el De re rustica de Columela ${ }^{13}$. A este propósito, conviene señalar que el análisis de las CNE que se lleva a cabo en este artículo sigue los principios teóricos propuestos por la Gramática Funcional (Dik $\left.1997^{2} \mathrm{ab}[1989]\right)^{14}$.

12 A este respecto, es importante tener en cuenta que, como señala Spevak $(2015,318)$, «la différence entre les arguments et les satellites adnominaux n'est alors pas seulement d'ordre sémantique et syntaxique (les arguments sont plus étroitement rattachés au nom que les satellites) mais encore d'ordre quantitatif».

13 Con el objetivo de extender el alcance de este estudio, se utilizan también pasajes de otras obras ubicadas en el mismo marco temporal (latín arcaico y clásico).

14 Este modelo teórico ha sido aplicado al latín particularmente por Pinkster (1984, 2015, 2021) y Baños (2009b), y tanto al griego antiguo como al latín por Torrego (1998), Baños et al. (2003) y Torrego et al. (2007). 
El trabajo tiene la estructura de los objetivos planteados más unas conclusiones.

\section{Otros elementos dentro de las Construcciones Nominalizadas de Evento: los disjuntos}

Para comenzar con los objetivos de este estudio, obsérvese la CNE encabezada por el nombre percursationem que presenta diversas modificaciones adjetivales ${ }^{15}$ :

(11) praeclaram illam percursationem tuam mense Aprili atque Maio, tum cum etiam Capuam coloniam deducere conatus es! («;Oh, aquella brillante gira tuya en los meses de abril y mayo, cuando intentaste fundar una colonia incluso en Capua!», Cic.Phil.2.100).

(11') tu percursauisti mense Aprili atque Maio.

Varios son los aspectos que cabe reseñar con respecto al tipo de complementos que acompañan al nombre percursationem en (11). En primer lugar, el pronombre posesivo tuam se identifica con el Sujeto de su verbo base percurro. En segundo, los ablativos mense Aprili atque Maio indican el momento en el que sucede la acción descrita por percursationem $\mathrm{y}$, por tanto, funcionan como adjuntos de Tiempo. En este sentido, es importante destacar que, como muestra el ejemplo (11), en su adaptación a la CNE estos elementos no conllevan transformaciones sintácticas, ya que no hay adjetivos derivados posibles y, por consiguiente, casi con total seguridad presentarían la misma codificación en su correspondiente predicación verbal base (11').

Más allá de la información sintáctica antes descrita, que básicamente se corresponde con la de una predicación verbal del tipo (11'), el pasaje de (11) es particularmente revelador por cuanto se refiere a los complementos illam y praeclaram.

Por una parte, el pronombre demostrativo illam tiene un uso exofórico, en la medida en que hace que la entidad -en este caso un evento- resulte identificable para el interlocutor sobre la base de su conocimiento del mundo en general (Pinkster 2015, 970). Específicamente, la información que aporta este pronombre, con respecto a toda la CNE de percursationem, es que la gira llevada a cabo por Marco Antonio -a quien va dirigida la acusación- resultaba bien conocida para el auditorio de Cicerón.

De forma relativamente similar, el adjetivo praeclaram 'brillante' contiene información sobre la predicación de percursationem en su conjunto, pues a través de él Cicerón, el emisor del enunciado, expresa su valoración -por cierto, marcadamente irónica- sobre la gira hecha por Marco Antonio en los meses de abril y mayo. En consecuencia, puede afirmarse que praeclaram asume en (11) la función propia atribuida a los disjuntos: «satellites that convey some form of comment by the speaker or writer either on the content or on the form and communicative function of the sentence (or clause) as a whole» (Pinkster 2015, 923).

Si se admite la interpretación dada a praeclaram en (11), puede concluirse que en sus construcciones los nombres de evento tienen la posibilidad de documentar o

Para una clasificación de los tipos de información (temporal, aspectual, modal, circunstancial, etc.) que pueden expresar los adjetivos que modifican un nombre predicativo, véase Gross (1996). 
desarrollar sintácticamente, además de argumentos y adjuntos, elementos que se tipificarían como disjuntos en la predicación verbal base. En vista de que los disjuntos no guardan relación alguna con la estructura argumental que heredan los nombres de evento a partir de sus verbos base, cabe preguntarse ahora cuáles son los contenidos semánticos que estos constituyentes son susceptibles de expresar dentro de las CNE.

\section{Tipo de contenido semántico expresado por los disjuntos}

De acuerdo con Pinkster $(2015,923)$, existen dos tipos de disjuntos en latín: por una parte, los que comentan el contenido de toda la predicación, denominados disjuntos de actitud (attitudinal disjuncts) y, por otra, aquellos que hacen comentarios sobre la forma y función comunicativa, los llamados disjuntos ilocutivos (illocutionary disjuncts).

Dentro de los disjuntos de actitud, es posible encontrar, a su vez, dos tipos de expresiones: i) las que indican el juicio o valoración subjetiva del hablante en relación con el contenido del enunciado y ii) las que indican en qué medida el hablante o escritor se responsabiliza de la veracidad del contenido del enunciado.

Por lo que respecta al corpus de análisis, a excepción de (17) - que se analiza más adelante-, todos los ejemplos documentados se identifican con el primer grupo de expresiones. Los pasajes de (12)-(14) se muestran como ejemplo:

(12) Tum partu Terra nefando Coeumque Iapetumque creat saeuomque Typhoea et coniuratos caelum rescindere fratres («Entonces en un abominable parto la Tierra creó a Ceo, a Iápeto, al cruel Tifón y a los hermanos conjurados para rasgar el cielo», Verg.Georg.1.278-280).

(13) Quin etiam uidemus ex acie efferri saepe saucios, et quidem rudem illum et inexercitatum quamuis leui ictu ploratus turpissimos edere («Más aún, frecuentemente vemos que los heridos son retirados del campo de batalla y que, ciertamente, aquel [soldado] inexperto y desentrenado emite muy vergonzosos lamentos por un golpe leve», Cic.Tusc.2.38).

(14) Nam quod scribis mirificam exspectationem esse mei neque ... («Pues por lo que me escribes de que hay una sorprendente expectación de mi persona y que no ...», Cic.Att.7.7.5).

En primer lugar, parece claro que el adjetivo nefando de (12) da cuenta de la valoración o percepción que tiene el emisor del mensaje sobre el evento referido por partu. Por su parte, en (13) cabe interpretar que el adjetivo en grado superlativo turpissimos manifiesta la opinión del emisor con respecto al evento que describe ploratus, nombre al que determina; así pues, parece que para Cicerón el hecho de que un soldado se lamente o llore por un golpe leve es un acto vergonzoso. Por último, en (14) puede entenderse que mirificam representa la opinión que, según Cicerón, tiene su corresponsal en relación con el estado o sentimiento de expectación general que hay sobre él (exspectationem mei). De acuerdo con las anteriores interpretaciones, los adjetivos nefando, turpissimos y mirificam se conciben en los ejemplos citados como "expresiones meta-comunicativas", en la medida que aportan información relevante para el acto de comunicación, no para las CNE en sí mismas. 
Al revisar el contenido semántico de estos adjetivos parece que todos ellos poseen una fuerte carga de subjetividad, sea porque describen una valoración altamente positiva (caso de mirificam) o marcadamente negativa (como nefando y turpissimos).

Pese a la relativa claridad que plantea el análisis de los ejemplos (12)-(14), se dan también casos en los que no es nada fácil establecer una diferencia clara entre los elementos que dentro de las CNE funcionan como disjuntos y los que lo hacen como adjuntos de Modo ${ }^{16}$; a manera de ejemplo, pueden observarse los pasajes (15) y (16):

(15) ... se regem, augentem bello Romanum imperium a proximis scelerata coniuratione pulsum ( «... él, siendo rey, cuando estaba engrandeciendo el imperio romano con la guerra, fue expulsado por sus allegados en una criminal conjuración», Liv.2.6.2).

(16) Montes effuso cursu Sabini petebant et pauci tenuere («Los sabinos intentaban alcanzar los montes corriendo en desbandada (lit.: con una carrera en desbandada, confusa) pero pocos los alcanzaron», Liv.1.37.4).

En el ejemplo (15), a priori el adjetivo scelerata puede entenderse como un disjunto que da cuenta del juicio del escritor sobre la conjuración (coniuratione), pero nada descarta que pueda interpretarse como un adjunto de Modo indicando que "la conjuración se hizo efectivamente de manera criminal", pues, por ejemplo, pudo llevarse a cabo mediante algún crimen. De forma relativamente similar, en (16) effuso parece funcionar como un disjunto con respecto al nombre de evento cursu, aunque también este adjetivo podría estar describiendo simplemente la forma confusa en la que los sabinos salieron corriendo. Como puede intuirse a partir de los ejemplos antes citados, la frontera entre la identificación de un disjunto o de una información adjunta de Modo no es siempre nítida y depende con frecuencia del contexto y del contenido semántico del adjetivo ${ }^{17}$.

En cuanto a los disjuntos de actitud que se relacionan con el valor de verdad del enunciado, existen dos subtipos: los llamados disjuntos de grado de verdad (degree of truth disjuncts) y los denominados disjuntos de limitación de verdad (qualified truth disjuncts). Dentro del corpus base, curiosamente solo se documenta un ejemplo donde parece plausible identificar la presencia, en una CNE, de un disjunto del primer tipo:

(17) Recurrendum est igitur ad qualitatium inter se dissidentium quasi quasdam

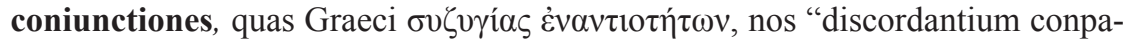

16 Aunque no hay criterios definitivos ni bien delimitados para la distinción entre los adverbios que funcionan como adjuntos y aquellos que lo hacen como disjuntos en el ámbito de las predicaciones verbales, a continuación se relacionan algunos de ellos: (i) «los disjuntos actitudinales no responden a una pregunta parcial sino total y suelen admitir una paráfrasis con la que se hace evidente que el adverbio expresa un juicio del hablante, no una modificación del predicado»; (ii) «suelen estar más alejados del verbo, generalmente en posición inicial o final, separados por una pausa, mientras que los adjuntos suelen aparecer más próximos al verbo» (Tarriño 2009, 364-365); y (iii) no son alcanzados por el ámbito de la negación (Pinkster 2004, 192). Otras pruebas empíricas para evaluar el funcionamiento de algunos adverbios y frases adverbiales en latín como disjuntos pueden verse en Pinkster (2004).

17 Pinkster $(2015,928)$ señala que «It must be said that formal criteria to distinguish this use of evaluative adverbs from their use as manner adjuncts so far have not yet been stablished». 
rationes" tolerabiliter dixerimus («Es necesario, por lo tanto, recurrir a ciertas mezclas, por así decirlo, de calidades opuestas entre sí, a las que los griegos

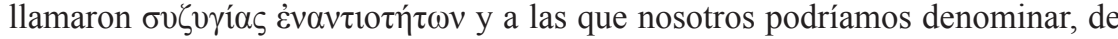
forma aceptable, "acoplamientos de cosas distintas"», Colum.2.2.3).

Como se puede ver, en (17) el nombre coniunctiones -con una interpretación eventiva apoyada por la presencia de qualitatium inter se dissidentium-se encuentra determinado por el cuantificador débil quasdam. Con respecto a este tipo de cuantificadores, según explica Pinkster $(2015,1109)$, con nombres abstractos quidam, dependiendo del contexto, puede intensificar o suavizar el significado del nombre. En su uso como "atenuante" puede aparecer combinado con quasi denotando "por decirlo de alguna manera, por así decirlo'. Es precisamente así que se entiende esta combinación en (17) y que cabe interpretarla como un disjunto de grado de verdad. En este caso en particular quasi quasdam presenta el contenido del nombre de evento coniunctiones como una expresión aproximada de lo que se quiere indicar, de modo que el grado del compromiso del hablante, Columela, en cuanto a la exactitud de esta expresión es más relativo. Esta relativa implicación de Columela además se ve reforzada por el comentario metalingüístico tolerabiliter dixerimus que, en la segunda oración de relativo, presenta la designación latina frente a la griega.

Por otra parte, en el corpus no se encuentran casos en los que una CNE documente un constituyente que sea susceptible de interpretarse como un disjunto ilocutivo, esto es, una expresión que, según se ha indicado más arriba, dé cuenta de la forma en la que el hablante invita al oyente a entender su enunciado. La pregunta que surge ahora es, pues, por qué estos componentes -que se documentan con relativa facilidad en las predicaciones verbales- sufren restricciones en su paso a las CNE en latín. En la siguiente sección intentaré responder a esta pregunta.

\section{Restricciones de los disjuntos en su paso a las Construcciones Nominalizadas de Evento}

Antes de comenzar, cabe tener presente dos aspectos: por una parte, como se ha dicho en $\S 3$, las posibilidades de codificación de los disjuntos dentro de las CNE se limitan a la presencia de adjetivos y material de concordancia nominal. Por otra, los disjuntos que se tratan en este apartado se refieren a la ilocución y las CNE no son ilocutivas, sino predicaciones que solo admiten valor de verdad; desde este enfoque, resulta ciertamente esperable que, si -como veremos- este tipo de disjuntos se expresan a través de predicaciones verbales, no cuenten con una derivación nominal.

Una vez dicho esto, parece oportuno ilustrar los diferentes tipos de contenidos que, según Pinkster $(2015,930)$, expresan los disjuntos ilocutivos en las predicaciones verbales; para ello se ofrecen los ejemplos (18)-(22):

(18) Ex iis igitur, ut supra diximus, si non ipsa membra genitalia conceptu atque fetu grauida ... legerimus, umbrae scilicet, non uendimiae laborauerimus («Así que si de estas [vides], como dijimos arriba, no hemos tomado los órganos reproductores mismos, preñados con el embrión concebido ... nos habremos inquietado evidentemente por la sombra, no por la vendimia», Colum.3.10.2). 
(19) Hoc ut scias, neutrum esse sine altero potest («Para que lo sepas, ninguna de las dos puede darse sin la otra», SEN.Epist.117.8).

(20) nunc mihi temporis eius quod mihi ad dicendum datur, quoniam in animo est causam omnem exponere, habenda ratio est diligenter («Ahora tendré que llevar escrupulosamente la cuenta del tiempo que se me ha dado para hablar, puesto que está en mi ánimo exponer toda la causa», Cic.Verr.1.32).

(21) et si quaeritis, ut ipse de me detraham, illos ego accusatores puto fuisse qui eiusmodi hominum furta odore aut aliquo leuiter presso uestigio persequebantur ( $(\mathbf{Y}$ si (me) lo preguntan, para quitarme yo mismo del medio, pienso que aquellos fueron unos acusadores que investigaban los robos de unos hombres de tal índole debido a su olfato o a alguna huella marcada levemente», Cic.Verr.4.53).

(22) Alter tibi descendit de Palatio et aedibus suis («El otro, hete aquí que baja desde su mansión del Palatino», Cic.S.Rosc.133).

En primer lugar, en el ejemplo (18), el disjunto ilocutivo ut supra diximus indica el estatus que tienen las palabras de Columela, el emisor, dentro del contexto que las rodea. Por su parte, el ejemplo (19), y más exactamente la oración introducida por ut, clarifica la intención que tiene Séneca al transmitir su enunciado. De forma un tanto diferente, la oración de quoniam en (20) se presenta como una justificación del hablante, Cicerón, a la declaración formulada; en este mismo sentido se puede interpretar la expresión et si quaeritis de (21), a través de la cual este mismo autor se pone en la situación del oyente para justificar lo que va a decir a continuación. Por último, en el ejemplo (22) el pronombre tibi funciona como un datiuus ethicus; a través de él se señala la persona que se encuentra más implicada afectivamente en la situación descrita y para la que esta se presenta como sorprendente o inesperada.

Excepto por el pronombre tibi de (22), todos los contenidos expresados por los disjuntos ilocutivos se desarrollan sintácticamente a través de oraciones (pseudo) subordinadas: una oración ilocutiva de modo con $u t(18)$, una pseudo-final introducida por $u t$ (19), una pseudo-causal con quoniam (20) o una pseudo-condicional con una prótasis introducida por si (21). Es precisamente la relativa complejidad sintáctica que entrañan estos disjuntos lo que podría explicar que no sean susceptibles de aparecer en una $\mathrm{CNE}$. A este respecto, hay que tener en cuenta que todas estas (pseudo) subordinadas tienen un alto grado de independencia con respecto a los enunciados (formados por la predicación nuclear más sus adjuntos) que comentan.

Para sustentar la anterior afirmación y siguiendo el trabajo de Lehmann (1988) sobre los tipos de conexión que se establecen entre oraciones, a continuación se clasifican los disjuntos de (18)-(21) en términos de autonomía (vs. integración), expansión ( $v s$. reducción) y aislamiento ( $v s$. unión).

En primer lugar, por lo que respecta a su grado de autonomía, las oraciones en cuestión presentan una baja degradación jerárquica (hierarchical downgrading), puesto que, a pesar de ser introducidas por medio de una conjunción subordinada (ut, quoniam, si) que las identifica como oraciones subordinadas, no tienen una función sintáctica con respecto al enunciado $-\mathrm{u}$ oración principal- $\mathrm{y}$ pueden aparecer incrustadas en él. Asimismo, tienen un alto nivel de autonomía; baste con considerar 
que trascienden el nivel representativo de la oración (se deben al acto comunicativo en sí) y que, en consecuencia, no se encuentran en el mismo plano sintáctico que las predicaciones nucleares con sus adjuntos.

En cuanto a su nivel de expansión, me centraré en la idea de que los disjuntos de (18)-(21) tienen un bajo grado de pérdida de características oracionales (desententialization) y, por tanto, de reducción; a manera de ejemplo se puede observar la construcción ut supra diximus de (18) que, entre otros aspectos, ostenta su propia fuerza ilocutiva (declarativa), no manifiesta restricciones relativas a su modo (indicativo), ni pérdida de la categoría de tiempo (perfecto).

Por último, de acuerdo con el criterio de aislamiento, las expresiones antes citadas evidencian un alto nivel de disjunción frente a las oraciones que comentan; nótese que, por ejemplo, no tienen por qué compartir el mismo predicado (cf. diximus, scias y quaeritis, en (18), (19) y (21), en relación con laborauerimus, potest y puto, respectivamente) ni los mismos participantes (cf. en (19), tu y hoc, complementos de la oración de disjunto, y neutrum, constituyente de la oración comentada).

Todos los argumentos anteriores ahondan en la idea del alto grado de independencia sintáctica y, por consiguiente, de complejidad que ostentan los disjuntos en relación con los enunciados que comentan. No parece, pues, de extrañar que tal complejidad no se pueda adaptar a la relativamente simple estructura nominal $-\mathrm{O}$ sintagmática- propia de las CNE, que deben expresar todo (argumentos, adjuntos y disjuntos) mediante básicamente nombres y adjetivos.

En cambio, los disjuntos de actitud, en concreto los de valoración del hablante, se expresan en las predicaciones verbales en muchas ocasiones a través de adverbios, como muestran optume y stulte en (23) y (24):

(23) Optume eccum incedit ad me («Estupendo, viene hacia mí», Plaut.Amph.335).

(24) Num stulte anteposuit exilii libertatem domesticae seruituti? («¿Antepuso estúpidamente la libertad del exilio a la esclavitud de su patria?», Cic.Tusc.5.109).

Dada la relación existente entre algunos adverbios y adjetivos no solo en términos morfológicos sino funcionales (Bos 1967, Haspelmath 2001, Villa 2006), es comprensible que se dé una fácil correspondencia sintáctica entre los disjuntos en las predicaciones verbales base y estos mismos elementos en las CNE.

Para concluir con esta sección conviene justificar, en la medida de lo posible, por qué ciertos dativos que funcionan como disjuntos dentro de las predicaciones verbales, sean ilocutivos, caso del datiuus ethicus tibi en (22), o de actitud (específicamente de verdad matizada), como el datiuus iudicantis mihi en (25), no se documentan en las estructuras nominalizadas de evento ${ }^{18}$.

(25) Verum edepol tua mihi odiosast amatio («Pero, por Pólux, tus amores me son odiosos», Plaut.Cas.328).

18 Frente a otros constituyentes que también pueden funcionar como disjuntos, caso de algunos sintagmas nominales (meo iudicio, mea [quidem] sententia) y preposicionales (cf., por ejemplo, aquellos encabezados por prae), me limito aquí a explicar estos dativos porque, en general, han gozado de un tratamiento particular en las gramáticas latinas. 
Por una parte, el datiuus ethicus refiere un participante que se encuentra involucrado de manera directa en el acto de habla (Maurel 1982); en este sentido, está limitado a pronombres personales de primera y segunda persona. Asimismo, se usa, por lo general, en contextos marcados, donde la situación que describe la oración resulta sorprendente o llamativa (Serbat 1996, 572) y donde los pronombres suelen estar en segunda posición y frecuentemente precedidos por una partícula o pronombre posesivo (Pinkster 2015, 931). Estas particularidades -ya en el propio nivel de la predicación verbal- quizá expliquen la falta de testimonios de este tipo de dativo en las CNE; además, a estos hechos habría que sumar que no siempre resulta fácil distinguir entre este empleo y el uso del dativo para marcar un beneficiario, de modo que la aparición de un datiuus ethicus en una CNE podría generar todavía más ambigüedades.

Por otra parte, el datiuus iudicantis especifica la persona a juicio de la cual resulta verdadero el enunciado. De forma menos restrictiva que el ethicus, con este tipo de dativo se documentan ejemplos como el del pronombre mihi en (25) y son frecuentes los casos con participios sustantivados y referencias topográficas (Kühner \& Stegmann 1912-1914, 321-322). En relación con el pasaje de (25), resulta oportuno señalar que los ejemplos que en algunas sintaxis (cf. Baños 2009a, 209; Pinkster 2015, 927) ilustran el empleo del datiuus iudicantis (fuera de aquellos con participios) son con el verbo sum. Tal circunstancia nos invita a pensar que si en contextos similares como los de (26) y (27) con los nombres de evento educatio y reprehensio apareciera un dativo con esta función, este se identificaría como un disjunto de toda la predicación y no solamente de los nombres de evento.

(26) Turturum educatio superuacua est, quoniam id genus in ornithone nec parit nec excudit («La cría de las tórtolas es inútil, ya que esta especie no pone huevos ni hace salir polluelos en una pajarera», CoLum.8.9.1).

(27) Reliquorum factorum eius, patres conscripti, difficilis est sane reprehensio et lubrica («La crítica de sus restantes actuaciones, senadores, es realmente difícil y peligrosa», Cic.Phil.2.59).

A pesar de que el datiuus iudicantis es el uso más típico del dativo como disjunto, su empleo tampoco está exento de confundirse con la expresión de un beneficiario; así pues, de nuevo su presencia en construcciones nominalizadas podría avocar a ambigüedades. Con todo, su falta de documentación en nuestro corpus no es un argumento suficiente para descartar por completo esta posibilidad, pero es indicativo.

En resumen, la ausencia de cierto tipo de disjuntos en las CNE en latín parece explicarse debido a dos factores generales: por una parte, la compleja codificación sintáctica que asumen en las predicaciones verbales - oraciones (pseudo) subordinadas- y, por otra, la ambigüedad que podría generar la aparición de un datiuus ethicus o iudicantis en el marco general de la predicación.

\section{Conclusiones}

A partir del desarrollo de este artículo se pueden extraer las siguientes conclusiones: 
En primer lugar, en latín las CNE pueden incluir no solo argumentos y adjuntos relacionados con los verbos base de los nombres de evento, sino que -como se ha demostrado- también documentan expresiones que sobrepasan el nivel representativo de la oración, esto es, disjuntos ( $\$ 2)$.

Dentro del corpus, estos disjuntos se relacionan particularmente con un contenido semántico: la manifestación de un juicio o valoración (sea positiva o negativa) del hablante sobre el contenido del enunciado. En este sentido, solo se ha documentado una expresión que parece indicar la medida en la que el hablante asume la responsabilidad de lo enunciado. Ambos contenidos pertenecen a la tipología de disjuntos de actitud y tanto en un caso como en el otro se codifican por medio de adjetivos (solos o modificados). De forma opuesta, ninguna CNE evidencia la presencia de disjuntos ilocutivos $(\S 3)$.

Asimismo, la restricción que supone la ausencia, de forma particular, de disjuntos ilocutivos en las CNE parece explicarse debido a que, en las predicaciones verbales, por lo general, se codifican a través de oraciones (pseudo) subordinadas que presentan un alto grado de independencia sintáctica con respecto a la oración que califican. En vista de tal complejidad sintáctica, resulta explicable que estos disjuntos no puedan adaptarse a la estructura -marcadamente más simple-de las CNE. Por cuanto se refiere a la no documentación de dativos como disjuntos (ethicus y iudicantis) en las $\mathrm{CNE}$, se ha argumentado a favor de que tal situación puede justificarse a causa de las ambigüedades que su aparición podría generar en el marco de la predicación $(\S 4)$.

Por otro lado, si se parte de la correspondencia que se da entre algunos adjetivos y adverbios, no es de extrañar que los disjuntos que más ampliamente se documentan en las CNE del corpus sean aquellos que se relacionan con la valoración del hablante y que se codifican mediante adjetivos, puesto que estos mismos elementos suelen expresarse a través de adverbios en las predicaciones verbales.

En última instancia, este artículo espera haber demostrado la importancia de seguir estudiando las construcciones nominalizadas, en vista de que su estudio puede contribuir a una mejor comprensión de la relación existente entre las categorías - tan aparentemente opuestas- Nombre y Verbo.

\section{Referencias bibliográficas}

Alexiadou, A. (2001), Functional Structure in Nominals: Nominalization and Ergativity, Ámsterdam/Filadelfia, John Benjamins.

Baños, J. M. et al. (2003) (eds.), Praedicativa I. Complementación en griego y latín, Santiago de Compostela, Universidad de Santiago de Compostela.

Baños, J. M. (2009a), «Dativo», en Baños, J. M. (coord.), Sintaxis del latín clásico, Madrid, Liceus, 185-209.

Baños, J. M. (2009b) (coord), Sintaxis del latín clásico, Madrid, Liceus.

Benveniste, E. (1975² [1984]), Noms d'agent et noms d'action en indo-européen, París, Librairie d'Amérique et d'Orient.

Bisetto, A. \& Melloni, C. (2007), «Result nominals: A lexical-Semantic Investigation», en Booij, G. et al. (eds.), On-Line Proceedings of the Fifth Mediterranean Morphology Meeting (MMM5), Boloña, Università degli Studi di Bologna, 393-412.

Boegel, T. (1902), De nomine verbali latino quaestiones grammaticae, Leipzig, Typis B. G. Teubneri. 
Bos, G. F. (1967), «L'adverbe en latin. Tentative de classification structurale», BSL 62, 106122.

Comrie, B. \& Thompson, S. (2007² [1985]), «Lexical Nominalization», en Shopen, T. (ed.), Language Typology and Syntactic Description. Vol. 3: Grammatical Categories and the Lexicon, Cambridge, Cambridge University Press, 334-381.

Dik, S. (1997²a [1989]), The Theory of Functional Grammar. Part 1: The Structure of the Clause, Berlín/Nueva York, Mouton de Gruyter.

Dik, S. (1997²b [1989]). The theory of Functional Grammar. Part 2: Complex and derived constructions, Berlín/Nueva York, Mouton de Gruyter.

Garzón, E. (2018), Nombres de Acción y otros derivados deverbativos en latín, Madrid, Universidad Autónoma de Madrid (Tesis doctoral).

Givón, T. (2001), Syntax. An Introduction. Vol. 2, Ámsterdam/Filadelfia, John Benjamins.

Grimshaw, J. (1990), Argument structure, Londres, MIT.

Gross, G. (1996), «Prédicats nominaux et compatibilité aspectuelle», Langages 121, 54-57.

Haspelmath, M. (2001), «Word Classes and Parts of Speech», en Smelser, N. \& Baltes, P. (eds.), International Encyclopedia of the Social and Behavioral Sciences, Oxford, Pergamon, 16538-16545.

Hopper, P. \& Thompson, S. (1985), «The iconicity of the universal categories 'noun' and 'verb'», en Haiman, J. (ed.), Iconicity in Syntax, Ámsterdam/Filadelfia, John Benjamins, 151-183.

Hout, A. van (1991), «Deverbal Nominalization, Object versus Event Denoting Nominals, Implications for Argument \& Event Structure», Linguistics in the Netherlands 8, 71-80.

Koptjevskaja-Tamm, M. (2012), Nominalizations, Londres/Nueva York, Routledge.

Kühner, R. \& Stegmann, C. (1912-1914), Ausführliche Grammatik der lateinischen Sprache, Hannover, Hahnsche Buchhandlung.

Lehmann, C. (1988), «Towards a typology of clause linkage», en Haiman, J. \& Thompson, A. (eds.), Clause combining in grammar and discourse, Ámsterdam/Filadelfia, John Benjamins, 181-225.

Lyons, J. (1977), Semantics. Vol. 2, Cambridge, Cambridge University Press.

Mackenzie, J. L. (1985), «Nominalization and valency reduction», en Bolkestein, M. et al. (eds.), Predicates and Terms in Functional Grammar, Dordrecht/Cinnaminson, Foris, 29-47.

Maurel, J. P. (1982), «Datifs libres et datifs liés en latin», Cahiers de Grammaire 4, 59-91.

Picallo, C. (1991), «Nominals and nominalization in Catalan», Probus 3 (3), 279-316.

Picallo, C. (1999), «La estructura del sintagma nominal: las nominalizaciones y otros sustantivos con complementos argumentales», en Bosque, I. \& Demonte, V. (eds.), Gramática descriptiva de la lengua española, Madrid, Espasa, 363-394.

Pinkster, H. (1984), Latijnse syntaxis en semantiek, Ámsterdam, B. R. Grüner Publishing.

Pinkster, H. (2004), «Attitudinal and illocutionary satellites in Latin», en Aertsen, H., Hannay, M. \& Lyall, R. (eds.), Words in their places. A Festschrift for J. Lachlan Mackenzie, Ámsterdam, Vrije Universiteit Amsterdam, 191-198.

Pinkster, H. (2015), The Oxford Latin Syntax. Vol. 1. The Simple clause, Oxford, Oxford University Press.

Pinkster, H. (2021), The Oxford Latin Syntax. Vol 2. The Complex Sentence and Discourse, Oxford, Oxford University Press.

Pultrová, L. (2011), The Latin Deverbative Nouns and Adjectives, Praga, Charles University in Prague, Karolinum Press.

Rosén, H. (1981), Studies in the Syntax of the Verbal Noun in Early Latin, Múnich, Wilhelm Fink Verlag. 
Rosén, H. (1983), «The Mechanisms of Latin Nominalization and Conceptualization in Historical View», en Haase, W. \& Temporini, H. (eds.), Aufstieg Und Niedergang Der Römischen Welt, Berlín/Nueva York, De Gruyter, 178-211.

Serbat, G. (1996), Grammaire fondamentale du latin VI. L'emploi des cas en latin. Vol. I: Nominatif, Vocatif, Accusatif, Génitif, Datif, Lovaina/París, Peeters.

Sleeman, P. \& Brito, A.M. (2010a), «Nominalization, event, aspect and argument structure: A syntactic approach», en Duguine, M., Huidobro, S. \& Madariaga, N. (eds.), Argument Structure and Syntactic Relations: A Cross-Linguistic Perspective, Ámsterdam/Filadelfia, John Benjamins, 113-129.

Sleeman, P. \& Brito, A. M. (2010b), «Aspect and argument structure of deverbal nominalizations: a split vP analysis», en Alexiadou, A. \& Rathert, M. (eds.), The Syntax of Nominalizations across Languages and Frameworks, Berlín/Nueva York, De Gruyter Mouton, 199-217.

Spevak, O. (2014), The Noun Phrase in Classical Latin Prose, Leiden/Boston, Brill.

Spevak, O. (2015), «Les noms verbaux en latin», BSL CX (1), 289-321.

Tarriño, E. (2009), «Adverbios y partículas», en Baños, J.M. (coord.), Sintaxis del latín clásico, Madrid, Liceus, 349-374.

Torrego, E. (1998) (ed.), Nombres y funciones. Estudios de sintaxis griega y latina, Madrid, Ediciones Clásicas-UAM.

Torrego, E. et al. (2007) (eds.), Praedicativa II. Esquemas de complementación verbal en griego antiguo y en latín, Zaragoza, Universidad de Zaragoza.

Villa, J. de la (2006), "Adverbs as a part of speech in Ancient Greek», en Crespo, E. et al. (eds.), Word Classes and Related Topics in Ancient Greek, Lovaina, Peeters, 405-439. 